\title{
Interference of circRNA HIPK3 alleviates cardiac dysfunction in lipopolysaccharide-induced mice models and apoptosis in $\mathrm{H} 9 \mathrm{C} 2$ cardiomyocytes
}

\author{
Shunyang Fan ${ }^{1 \#}$, Kailun Hu ${ }^{1 \#}$, Deyin Zhang ${ }^{2}$, Fuyun Liu ${ }^{3}$ \\ ${ }^{1}$ Central Department of Cardiology, ${ }^{2}$ Department of Breast Surgery, ${ }^{3}$ Department of Pediatric Orthopaedics, The Third Affiliated Hospital of \\ Zhengzhou University, Zhengzhou, China \\ Contributions: (I) Conception and design: S Fan, F Liu; (II) Administrative support: F Liu, K Hu; (III) Provision of study materials or patients: K Hu, \\ D Zhang; (IV) Collection and assembly of data: S Fan, K Hu; (V) Data analysis and interpretation: S Fan, D Zhang; (VI) Manuscript writing: All \\ authors; (VII) Final approval of manuscript: All authors. \\ "These authors contributed equally to this work. \\ Correspondence to: Fuyun Liu. Department of Pediatric Orthopaedics, The Third Affiliated Hospital of Zhengzhou University, Zhengzhou, China. \\ Email: fuyunliuzzdx3@sina.com.
}

Background: Circular RNAs (circRNAs) have been deemed to be microRNA (miRNA) sponges that are involved in multiple biological processes. It has not yet been corroborated whether the regulation of circular RNA HIPK3 (circHIPK3) can be used for the treatment of myocardial dysfunction.

Methods: In this study, we aimed to investigate the cardioprotective effects and apoptosis inhibition of circHIPK3 regulation on lipopolysaccharide (LPS)-induced myocarditis in vivo and vitro. C57BL/6 mice were exposed to LPS with or without knockdown of circHIPK3. Reverse transcription polymerase chain reaction (RT-PCR) testing was used to evaluate the expression of circHIPK3. Hematoxylin and eosin (HE) staining, immunohistochemistry (IHC), Cell Counting Kit-8 (CCK8), flow cytometry, terminal deoxynucleotidyl transferase dUTP nick end labeling (TUNEL) assay, enzyme-linked immunosorbent assay (ELISA), and western blotting were used to evaluate histopathology, proliferation, apoptosis, oxidative stress, and inflammatory response, respectively. Cardiac function and myocardial damage were also evaluated.

Results: It was proven that short hairpin RNA1 (shRNA1) was a superior interference of circHIPK3. The results revealed that knockdown of circHIPK3 effectively alleviated myocardial tissue damage, improved cardiac function, and suppressed cardiomyocyte apoptosis in the animal model of LPS-induced myocarditis. Furthermore, LPS-induced oxidative injuries and inflammation in the myocardium were also partly reversed after circHIPK3 knockdown. In vitro, being LPS-induced enhanced the levels of heart damage markers, simultaneously inhibited proliferation, promoted apoptosis, and stimulated oxidative stress and inflammation of H9C2 cells. Fortunately, the abnormalities mentioned were partly reversed following circHIPK3 knockdown.

Conclusions: In this study, we characterized the expression and regulation of circHIPK3 in LPS-induced myocarditis in the animal model and H9c2 cells. The results demonstrated that circHIPK3 expression is significantly upregulated when exposed to LPS in vivo and in vitro. Knockdown of circHIPK3 effectively alleviated LPS-induced myocarditis.

Keywords: Lipopolysaccharide-induced myocarditis (LPS-induced myocarditis); circular RNAs (circRNAs); cardiomyocyte apoptosis; C57BL/6 mice; cardioprotective effects

Submitted Jun 29, 2020. Accepted for publication Aug 25, 2020.

doi: 10.21037/atm-20-5306

View this article at: http://dx.doi.org/10.21037/atm-20-5306 


\section{Introduction}

Myocardial dysfunction, one kind of common cardiac dysfunction, is life-threatening, and difficult to treat due to the proliferation of inflammatory lesions in the myocardium (1). Myocardial dysfunction can result from a wide spectrum of infectious pathogens, including viruses, chlamydia, bacteria, protozoans, rickettsia, and fungi, it can also result from toxic and hypersensitivity reactions (2,3). Myocardial dysfunction seriously endangers health, in severe cases patients may suffer from heart failure or even sudden death (4). Currently, the treatment of myocardial dysfunction includes intravenous immunoglobulin, medications, implantable cardioverter defibrillator, heart transplant, and biotherapy (5). More effective treatment strategies or ideas for common cardiac dysfunction are in studing.

Circular RNAs (circRNAs) are a novel class of noncoding RNAs, characterized by covalently closed loop structures with neither 5 ' to 3 ' polarity nor a polyadenylated tail (6). They are produced by precursor messenger RNA (mRNA) back-splicing and are extensively expressed in mammals with highly conserved, stable, and tissue-specific patterns (7). There is an increasing amount of evidence indicating that circRNAs are involved in various diseases, such as cardiac fibrosis (8), diabetes (9), and carcinomas (10).

Growing evidence has implicated circRNA in the pathogenesis of multiple cardiac dysfunction diseases (11). Recently, circRNAs have attracted attention in treatment of myocardial dysfunction, due to being upregulated in myocarditis patients. Cai et al. reported that circ-Ttc3 exert the protection effect on cardiac function after myocardial infarction by sponging miR-15b (12). Zhang et al. reported that circRNAs play substantial roles in pediatric fulminant myocardial dysfunction and hascirc-0071542 may be a promising biomarker of myocardial dysfunction (13). Further, the results of functional analysis demonstrated that differentially expressed circRNAs mainly contributed to inflammation and immunity. Silencing circANKRD36 exerted an anti-inflammatory and anti-apoptosis function in $\mathrm{H} 9 \mathrm{c} 2$ cells when exposed to lipopolysaccharide (LPS) via the p38MAPK/NF$\kappa \mathrm{B}$ pathway, and up-regulation of miR-138 (14). Several cellular processes were identified in which circRNAs had important roles. Wang et al. found that circRNA mediates cardiomyocyte death via microRNA (miRNA)dependent upregulation of MTP18 expression (15). A previous study demonstrated that circRNAs mediate essential physiological and pathological processes in the cardiovascular system (16). Homeodomain-interacting protein kinase 3 (HIPK3) had a high expression level in the heart, which was associated with transcription regulation, steroidogenic gene expression, and apoptosis (17). Previous studies have shown that circHIPK3 was related to osteosarcoma, gallbladder cancer, and lens epithelial cells $(18,19)$. However, the unique relationship between circHIPK 3 and cardiac dysfunction remains unclear.

In this study, we found that circHIPK3 can be upregulated significantly in LPS-induced myocarditis animal models and H9c2 cells. Knockdown of circHIPK3 effectively alleviated myocardial tissue damage, improved cardiac function, and suppressed cardiomyocyte apoptosis in vivo. In vitro, knockdown of circHIPK 3 can reverse the abnormalities mentioned induced by LPS, such as high levels of heart damage markers, proliferation inhibition, apoptosis, oxidative stress, and inflammation of $\mathrm{H9C} 2$ cells.

We present the following article in accordance with the ARRIVE reporting checklist (available at http://dx.doi. org/10.21037/atm-20-5306).

\section{Methods}

\section{Acquisition of lentivirus and cell transfection}

The lentivirus-containing short hairpin RNA (shRNA) targeting circHIPK3 was obtained from GenePharma (Shanghai, China), and the pLenti-GIII-CMV vector for circHIPK3 overexpression was obtained from Applied Biological Materials (ABM, Richmond, BC, Canada).

All shRNAs were transfected into the H9C2 cell lines. At $48 \mathrm{~h}$ post-transfection, the cells were selected with puromycin $(2 \mu \mathrm{g} / \mathrm{mL})$ for 2 weeks to construct cell lines with stable circHIPK3 knockdown or overexpression. The efficiency of transfection was verified by reverse transcription polymerase chain reaction (RT-PCR). The H9C2 cells were transfected with the abovementioned oligonucleotides and plasmids using Lipofectamine 3000/ Invitrogen (Thermo-Fisher, Whaltham, MA, USA) according to the manufacturer's instructions.

\section{Animal models}

All animal experiments were approved by the Animal Care and Use Committee of the Third Affiliated Hospital of Zhengzhou University (Zhengzhou, Henan, China) and 
conducted according to the declaration of the $8^{\text {th }}$ edition of the Guide for the Care and Use of Laboratory Animals (2011).

C57BL/6 mice were purchased from the Third Affiliated Hospital of Zhengzhou University (Zhengzhou, Henan, China). All mice were provided with a specific-pathogenfree room $\left(25^{\circ} \mathrm{C}, 65 \%\right.$ humidity) and exposed to a $12 \mathrm{~h}$ light-dark cycle; mice were fed standard chow and drinking water freely. After acclimatizing for at least $1 \mathrm{wk}$, the mice were manipulated with LPS intervention, dosages of LPS were based on previous studies (20). After $2 \mathrm{~h}$ of LPS induction, the mice received cell transfection, approximately $1.5 \mu \mathrm{L}\left(1 \times 10^{12} \mu \mathrm{g} / \mathrm{mL}\right)$ of adeno-associated virus containing circHIPK3-small hairpin RNAs [shRNAs: short hairpin RNA1 (shRNA1), shRNA2, shRNA3] were used. RTPCR was used to detect expression of circHIPK3. After $24 \mathrm{~h}$, the mice were anesthetized by pentobarbital sodium (50 $\mathrm{mg} / \mathrm{kg}$, ip), and ultrasound M-mode echocardiography was immediately conducted to evaluate cardiac function [including heart rate (HR), left ventricular wall thickness (LVWT), the left ventricle ejection fraction (LVEF) and left ventricular end-systolic volume (LVESV)] using a vevo 770 ultrasound machine (Visual Sonics, Toronto, Canada). Laparotomy and thoracotomy were then performed before blood samples were taken from the inferior vena cava and centrifuged for $10 \mathrm{~min}$ at $1,000 \mathrm{~g}$, and the supernatant plasma was collected for subsequent testing. The hearts of the mice were also obtained for the following experiments.

\section{Cell Counting Kit-8 (CCK8)}

We created four groups of H9c2 cells: (I) control group, (II) LPS induced group, (III) LPS + shRNA-NC group, and (IV) LPS + circHIPK3-shRNA1 group. Each group of H9c2 cells was seeded in a 96-well plate with $5 \times 10^{3}$ cells/ well. Cell viability was estimated by a CCK-8 (Dojindo, Kumamoto, Japan) according to the manufacturer's instructions. Briefly, the CCK- 8 solution was added to each well and incubated at $37^{\circ} \mathrm{C}$ for $1 \mathrm{~h}$. The absorbance at $450 \mathrm{~nm}$ was detected using a microplate reader (Thermo Fisher, Whaltham, MA, USA). Cell viability was calculated by (experimental group absorbance value/control group absorbance value) $\times 100 \%$.

\section{Histology}

Pathological damage of myocardial tissue in each group of mice was detected by hematoxylin and eosin (H\&E) staining. Collected myocardial tissue was fixed in $4 \%$ paraformaldehyde for $24 \mathrm{~h}$, embedded in paraffin, and sectioned at a thickness of about $4 \mu \mathrm{m}$. The slices were stained with $\mathrm{H} \& \mathrm{E}$ and toluidine blue, and were then placed under light microscopy to view pathological changes of the tissues. Five visual fields were taken randomly for each slice.

\section{Immunobistochemistry (IHC)}

IHC assay was used to detecte the content of IL-6. The assay was performed as previously described by McGuire et al. (21). Paraffin sections were separated in xylene and rehydrated in gradient ethanol (absolute ethanol, 95\% ethanol, 70\% ethanol and 50\% ethanol). After the antigen was extracted in a $10 \mathrm{mM}$ citric acid buffer, the tissue sections were incubated in $3 \% \mathrm{H}_{2} \mathrm{O}_{2}$ for $10 \mathrm{~min}$, and sealed at room temperature for $1 \mathrm{~h}$. The tissue sections were then incubated overnight with anti-IL-6 (ab208113, 1:50). Sections were then washed with TBST and incubated with SignalStain ${ }^{\circledR}$ Boost IHC Detection Reagent (CST, Danvers, MA, USA) for $30 \mathrm{~min}$ at room temperature. After that, sections were stained with a SignalStain ${ }^{\circledR}$ DAB Substrate Kit (CST, Danvers, MA, USA) and were observed using a FluoView FV1000 confocal microscope (Olympus, Shinjuku, Tokyo, Japan). All experiments were performed in triplicate.

\section{Reactive oxygen species (ROS) generation}

ROS generation was measured following previous report (22). Firstly, the tissue was incubated in Hank's balanced salt solution with $5 \mathrm{mM} \mathrm{H}$ 2DCFDA for $1 \mathrm{~h}$. Upon oxidation, the nonfluorescent H2DCFDA was converted to the highly fluorescent 2',7'-dichlorofluorescein (DCF). The fluorescence of DCF was detected at an excitation wavelength of $488 \mathrm{~nm}$ and an emission wavelength of 515-540 nm using an Olympus confocal microscope. Cells images were also obtained by digital interference contrast (DIC). The mean fluorescence intensity of each cell and the number of ROS-positive cells per field were analyzed using Image J software.

\section{Enzyme-linked immunosorbent assay (ELISA)}

The levels of cardiac troponin (cTnI), creatine kinase myocardial band (CK-MB), and myoglobin $(\mathrm{Mb})$ in myocardial tissue and $\mathrm{H} 9 \mathrm{c} 2$ cells were determined with ELISA kits [Neobioscience (Shenzhen, GD, China) and Cloud-Clone (Wuhan, Hubei, China)] following the 
manufacturer's protocols. The levels of inflammatory factors (TNF- $\alpha$, iNOS and IL-10) in peripheral blood, as well as IL-10 and IL-6 in H9c2 cells were also determined using ELISA kits (NeoBioscience and Cloud-Clone) following the manufacturer's protocols. The contents of lactate dehydrogenase (LDH), superoxide dismutase (SOD), and malondialdegyde (MDA) were measured using kits strictly according to the instructions. These kits were purchased from Shanghai Qiaoyu Biotechnology Co., Ltd. (Shanghai, China).

\section{Terminal deoxynucleotidyl transferase dUTP nick end (TUNEL) assay}

The prepared paraffin sections were dewaxed, rehydrated, and washed. Then, the sections were incubated with TUNEL reaction mixture (Roche Diagnostics, Indianapolis, IN, USA) according to the instructions of manufacturer. Apoptosis cells were detected by fluorescence microscopy (Olympus, Shinjuku, Tokyo, Japan).

\section{Flow cytometry}

Apoptosis of $\mathrm{H} 9 \mathrm{c} 2$ cells was measured by flow cytometry. Cells were prepared as previously reported (23). Annexin V-FITC (fluorescein isothiocyanate) staining was used to evaluate the apoptotic cells, and propidium iodine (PI) exclusion method was used to detect any dead cells. Then cells were analyzed using flow cytometry FACSCalibur instrument (BD Biosciences, NJ, USA) according to the manufacturer's instructions.

\section{Real-time PCR}

The relative expression levels of circHIPK3 were extracted using the TRIzol reagent (Invitrogen, Waltham, MA, USA) and reverse transcription was carried out using a RT-PCR kit (TaKaRa, Shiga, Japan). Real-time PCR was performed with SYBRExScript ${ }^{\mathrm{TM}}$ RT-PCR Kit (TaKaRa, Shiga, Japan) on an iQ5 Multicolor Real-Time PCR Detection System (Bio-Rad, Hercules, CA, USA) according to the manufacturer's protocol. The comparative $2^{-\triangle \Delta C T}$ method was used to analyze the relative expression of circHIPK3.

\section{Western blot analysis}

Myocardial tissues $(0.1 \mathrm{~g})$ were collected from each group of mice and were homogenized in $1 \mathrm{~mL}$ of protein extraction buffer. The supernatant was collected following centrifugation and the protein concentrations were detected using the bicinchonic acid (BCA) protein assay kit. The quantity of total protein samples was $20 \mu \mathrm{g}$, which was loaded into $10 \%$ sodium dodecyl sulfate polyacrylamide gel electrophoresis (SDS PAGE) loading buffer and subsequently transferred to polyvinylidene difluoride (PVDF) membranes. The PVDF membranes were sealed with $5 \%$ skimmed milk at $37{ }^{\circ} \mathrm{C}$ for $2 \mathrm{~h}$. Then, they were incubated overnight at $4{ }^{\circ} \mathrm{C}$ with the following primary antibodies: Bax (ab32503, 1:1,000, Abcam, UK), Bcl-2 (ab692, 1:500, Abcam, UK), caspase-3 (ab13847, 1:500, Abcam, UK), and caspase-9 (ab32539, 1:1,000, Abcam, UK). Subsequently, PVDF membranes were incubated for $60 \mathrm{~min}$ at $37^{\circ} \mathrm{C}$ with goat anti-rabbit IgG horseradish peroxidase (HRP) conjugated secondary antibodies (Zhuangzhi Biotechnology, Xi'an, China). Band densities were determined and analyzed with an automatic digital gel image analysis system Bio-Rad CFX-96 (Bio-Rad, CA, USA).

\section{Statistical analysis}

Data are shown as the mean \pm SD. Statistical analysis of the data were performed using the GraphPad Prism 5.0 statistical program (San Diego, CA, USA). Two groups were compared using the Student's $t$-test. Multiple groups were performed by one-way analysis of variance (ANOVA), followed by a least significant difference (LSD) test.

\section{Results}

\section{CircHIPK3 interference and influence of knockdown of circHIPK3 on cardiac function in vivo}

In order to screen the shRNA with superior interference efficiency, three kinds shRNAs were used, these were shRNA1, shRNA2, and shRNA3. RT-PCR was used to detect the relative miRNA expression level of circHIPK3. As displayed in Figure 1A, compared with the control group, shRNA1 significantly exerted interference efficiency. As shown in Figure 1B, the sample being LPS-induced significantly up-regulated relative miRNA expression level of circHIPK3; however, shRNA1 down-regulated the level. Results of H\&E staining showed that being LPS-induced caused serious tissue damage compared with the control group. Notably, participation of shRNA1 reversed these 
A

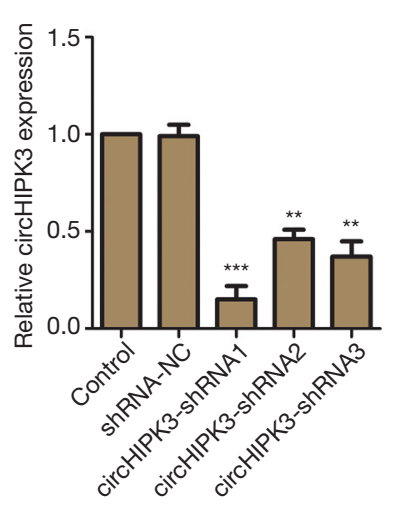

D

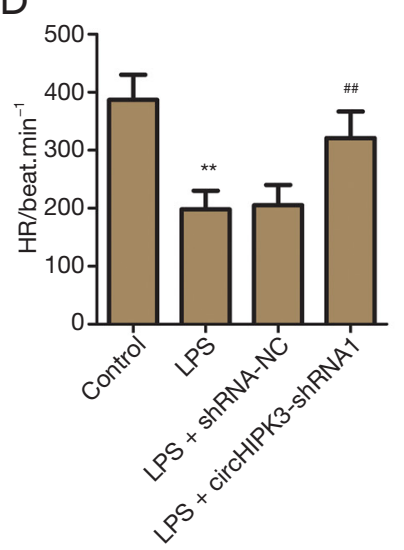

B
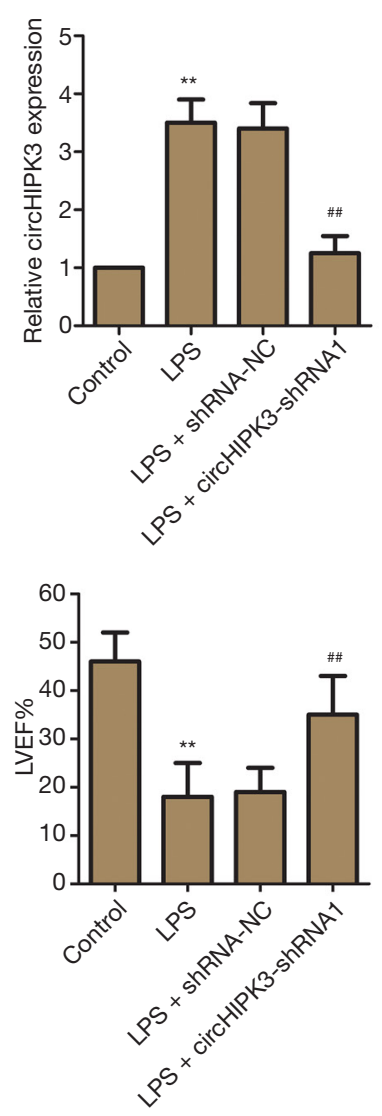

C
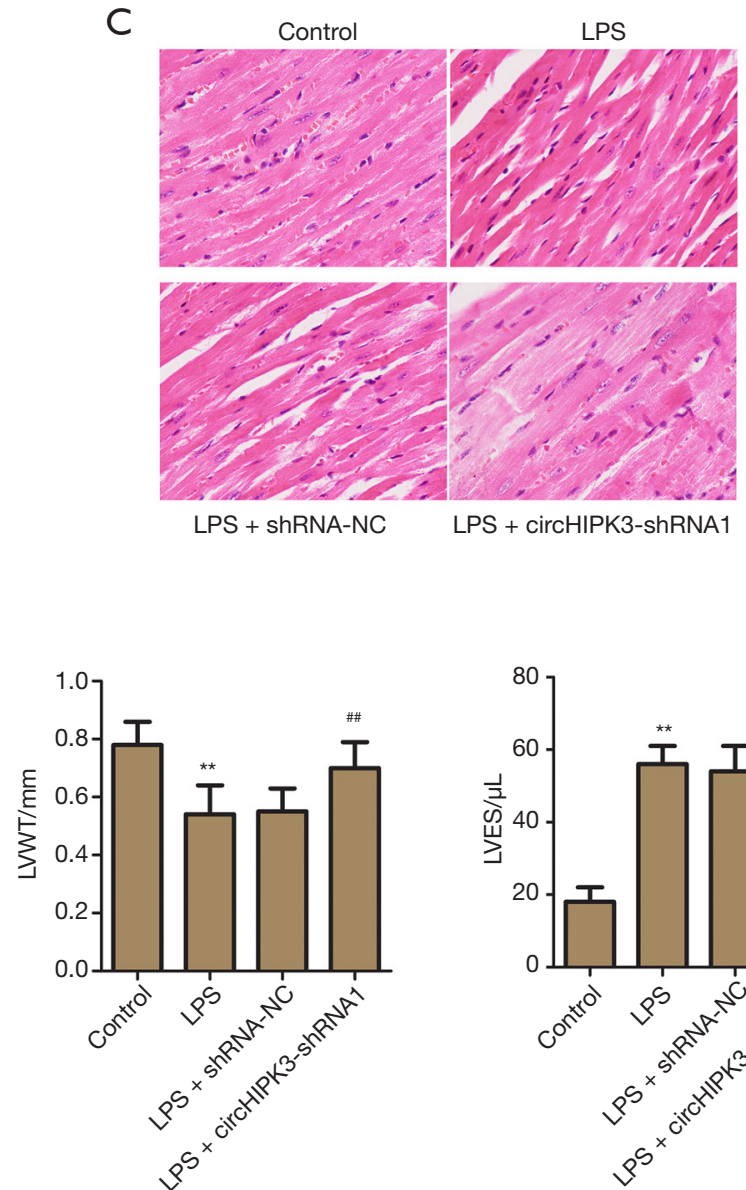

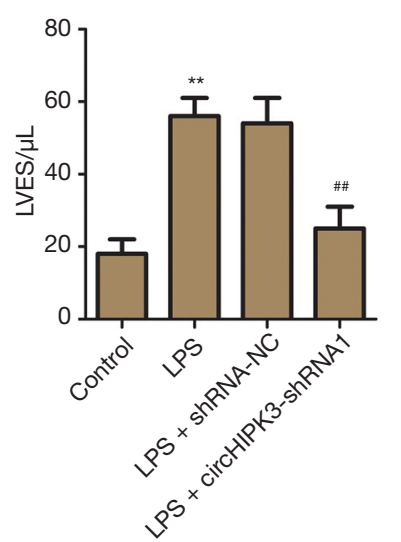

Figure 1 Circular RNA HIPK3 (CircHIPK3) interference, and influence of knockdown of circHIPK3 on cardiac function in vivo. (A) Selection of short hairpin RNAs (shRNAs) that were used to interfere with circHIPK3. (B) Relative circHIPK3 expression in tissue. (C) Representative pictures of hematoxylin and eosin (H\&E) staining (magnification $\times 200$ ). (D) Levels of cardiac function index: heart rate (HR), left ventricular wall thickness (LVWT), left ventricular ejection fraction (LVEF) and left ventricular end systolic volume (LVESV). The results are presented as mean $\pm \mathrm{SD}$ and represent three individual experiments [ ${ }^{* *}, \mathrm{P}<0.01,{ }^{* * *}, \mathrm{P}<0.001$ versus control group; ${ }^{\# \#}, \mathrm{P}<0.01$ versus lipopolysaccharide (LPS)-induced model group].

damages (Figure 1C). As shown in Figure 1D, participation of shRNA1 enhanced the level of HR, LVWT, and LVEF, as well as reduced the level of LVESV, which indicated that shRNA1 interfered with circHIPK3 effectively and further exerted a cardioprotective function. Therefore, we selected siRNA1 for subsequent experiments as it was found to be the most effective siRNA.

\section{Effect of circHIPK3 knockdown on beart damage markers and myocardial apoptosis in vivo}

ELISA was used to detect heart damage markers in serum. As in Figure 2A, knockdown of circHIPK3 significantly reduced the expression levels of cTnI, CK-MB, and $\mathrm{Mb}$, which were enhanced by being LPS-induced. As shown in Figure 2B, the results of TUNEL assay suggested that knockdown of circHIPK3 significantly decreased the percentage of apoptosis cells, which was increased by being LPS-induced. Western Blot assay was used to detect the relative protein level of caspase-3, caspase-9, Bax and Bcl-2. As shown in Figure 2C, knockdown of circHIPK3 significantly decreased the ratio of $\mathrm{Bax} / \mathrm{Bcl}-2$, cleaved caspase- $9 /$ caspase- 9 , and cleaved caspase-3/caspase-3. These results demonstrated that knockdown of circHIPK3 suppressed heart damage and cardiomyocyte apoptosis. 
A

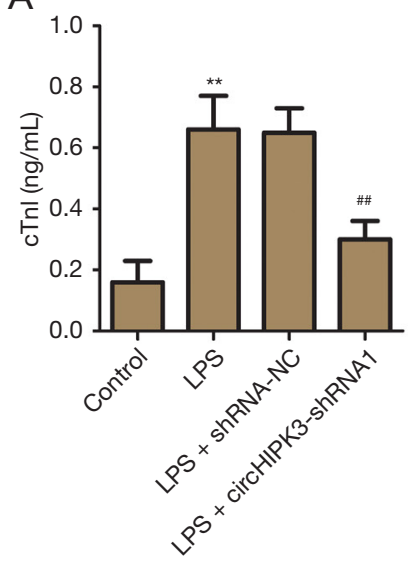

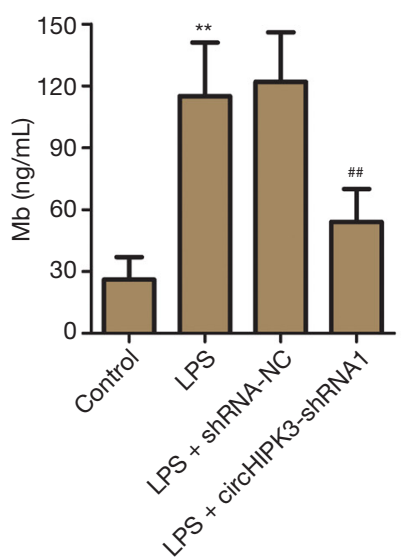

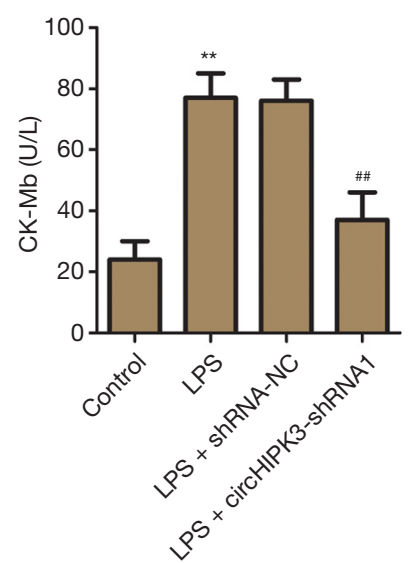

B
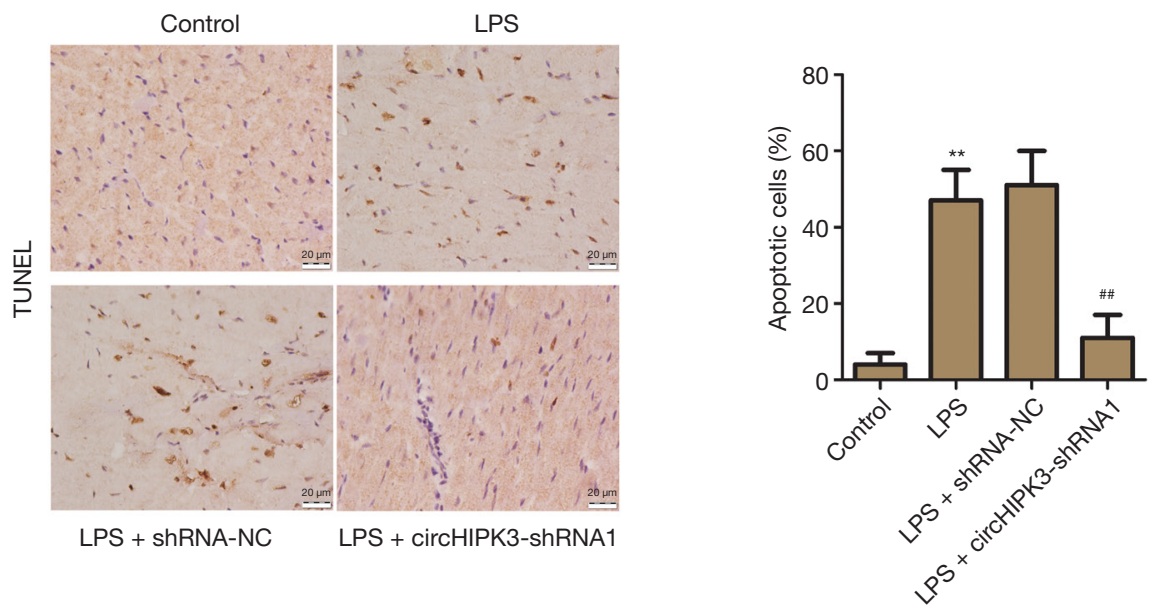

C
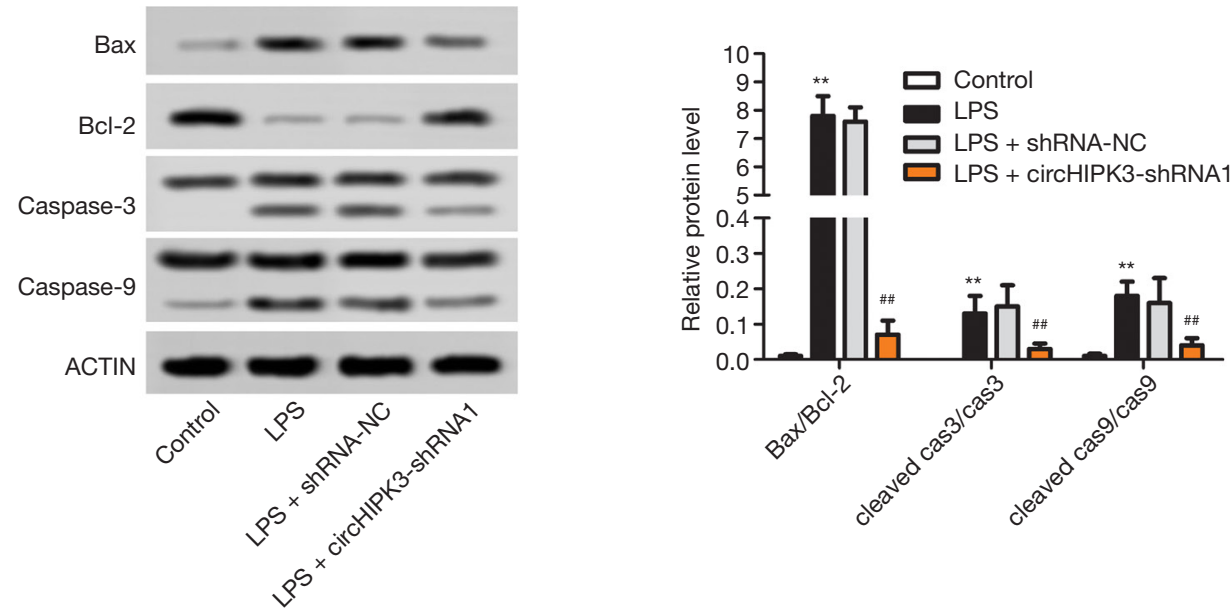

Figure 2 Effect of circHIPK3 knockdown on heart damage markers and myocardial apoptosis in vivo. (A) Levels of heart damage markers in serum: cardiac troponin $1(\mathrm{cTnI})$, creatine kinase myocardial band (CK-MB), and myoglobin (Mb). (B) Effect of circHIPK3 knockdown on myocardial apoptosis (magnification $\times 200$ ). (C) Representative pictures of Western Blot assay and relative protein level of Bax/Bcl-2, cleaved caspase-9/caspase-9, and cleaved caspase- 3 /caspase- 3 . The results are presented as mean $\pm \mathrm{SD}$ and represent three individual experiments ${ }^{* *}$, $\mathrm{P}<0.01$ versus control group; ${ }^{\# \#, ~} \mathrm{P}<0.01$ versus lipopolysaccharide (LPS)-induced model group]. 


\section{Effect of circHIPK3 knockdown on oxidative stress and inflammation in vivo}

The effects of knockdown of circHIPK3 on oxidative stress and inflammation were investigated. Numerous findings have indicated that oxidative stress is an important pathogenic driving force of myocardial damage (24). In Figure $3 A, B$, the levels of MDA, LDH and ROS were enhanced by LPS-induction, concomitantly, the level of SOD was decreased. Fortunately, knockdown of circHIPK3 partly reversed these effects. Inflammatory factors in peripheral blood were detected by ELISA. As shown in Figure 3C, being LPS-induced significantly increased the levels of TNF- $\alpha$ and iNOS, and slightly enhanced the IL-10 level. Notably, knockdown of circHIPK3 partly reversed the improvement of TNF- $\alpha$ and iNOS levels, and enhanced the level of IL-10. As in Figure 3D, the results of the IHC experiment showed that being induced with LPS enhanced the percentage of IL-6 positive cells compared with the control group, and that fortunately, knockdown of circHIPK3 partly reversed this trend. Collectively, these results suggested that knockdown of circHIPK3 effectively alleviated oxidative stress and inflammation in vivo.

\section{Effect of circHIPK3 knockdown on beart damage markers in $\mathrm{H} 9 \mathrm{C} 2$ cells}

We selected H9C2 cells for in vitro experiments. In Figure $4 A$, relative miRNA expression level of circHIPK3 was increased by being LPS-induced, while shRNA1 application partly reversed this trend. As shown in Figure 4B,C,D, knockdown of circHIPK3 decreased the levels of heart damage markers, including cTnI, CK$M B$ and $M b$. These findings demonstrated that shRNA1 effectively interfered with circHIPK3 expression in vitro, further, knockdown of circHIPK3 played the role of a myocardial cell protector. These results were consistent with the results of experiments in vivo.

\section{Effects of circHIPK3 knockdown on proliferation, apoptosis, oxidative stress, and inflammation of $\mathrm{H} 9 \mathrm{C} 2$ cells}

According to the results of the CCK8 assay, after exposure to LPS, the proliferation of cells was significantly reduced, while this trend was partly reversed by circHIPK3 knockdown (Figure 5A). As displayed in Figure 5B, the percentage of apoptotic cells was enhanced after being exposed to LPS, while knockdown of circRHIPK3 effectively reversed this trend. Correspondingly, being LPS-induced enhanced the ratio of $\mathrm{Bax} / \mathrm{Bcl}-2$, and knockdown of circHIPK3 effectively decreased the ratio (Figure 5C). Compared with the LPS-induced group, knockdown of circHIPK3 effectively increased the level of SOD and decreased the level of MDA (Figure 5D). As shown in Figure 5E, compared with the LPS-induced group, knockdown of circHIPK3 effectively increased the level of IL-10 and decreased the level of IL-6. Collectively, these results indicated that knockdown of circHIPK3 effectively expedited proliferation of $\mathrm{H} 9 \mathrm{C} 2$ cells, inhibited apoptosis of $\mathrm{H} 9 \mathrm{C} 2$ cells, and suppressed oxidative stress and inflammation in the in vitro experiment. Notably, these results were consistent with the results of the experiments in vivo.

\section{Discussion}

Myocardial dysfunction refers to the clinical and histological manifestations of a wide range of pathological immune processes in the heart (25). Although there is already a large body of research focused on the pathology of myocardial dysfunction, the medical treatment of myocardial dysfunction still requires significant progress. Thus, it is urgent to discover novel drug targets for myocardial dysfunction therapy.

The circRNAs are an emerging class of non-coding RNAs that are endogenous, abundant, and stable, formed by back-splicing events. It is reported that circRNAs play important roles in various diseases, such as colorectal cancer and pancreatic ductal adenocarcinoma $(26,27)$. An increasing body of evidence has suggested that circRNAs could be treated as diagnostic biomarkers and therapeutic targets for multiple diseases. Has-circ-0004277 is an outstanding biomarker for acute myeloid leukemia (28). Has-circ-0013958 may be a potential new biomarker of lung adenocarcinoma (29). In this current study, circHIPK3 was consistently enriched in the tissues of LPS-induced myocarditis mice models. Thus circHIPK3 can be used for new diagnostic and treatment strategies for myocarditis.

The main features of viral myocarditis are myocardial contraction and diastolic dysfunction; HR, LVWT, LVEF, and LVESV are commonly used to evaluate myocardial contractile and diastolic function. The data of the echocardiography experiment showed that participation of shRNA1 enhanced the level of HR, LVWT, and LVEF while it reduced the level of LVESV, which indicated that knockdown of circHIPK3 exerted the heart protective 
A
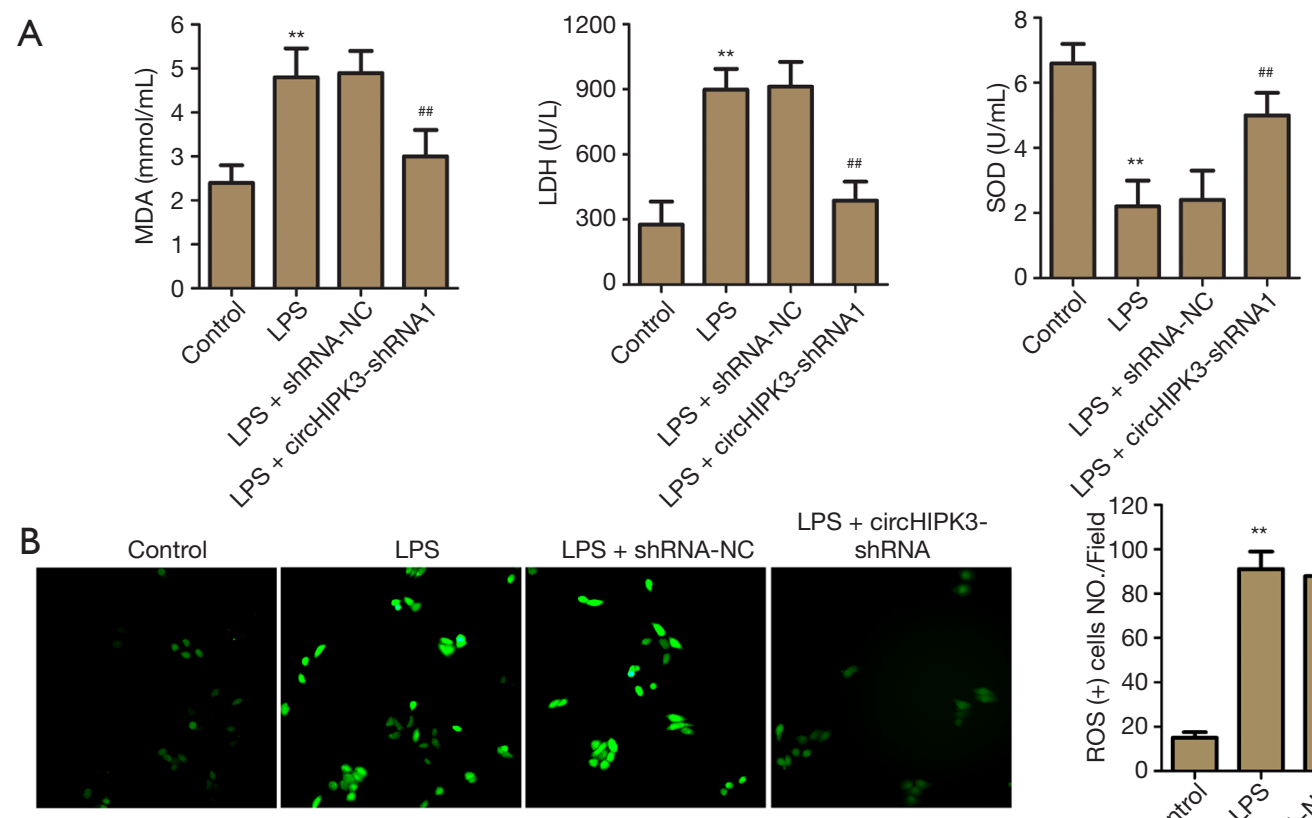

LPS + circHIPK3-
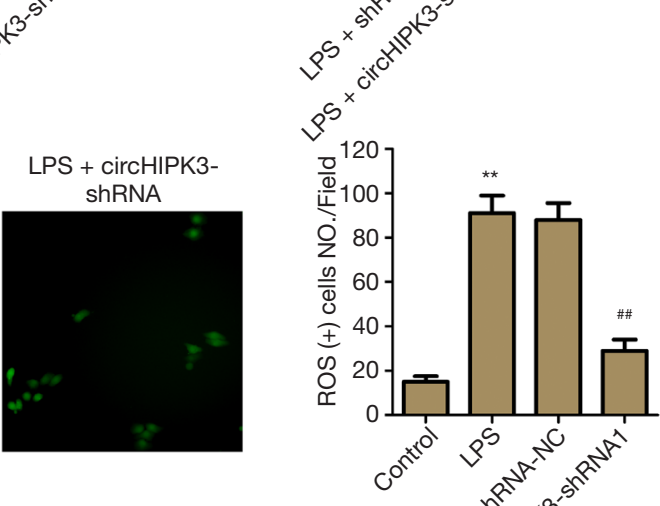

C
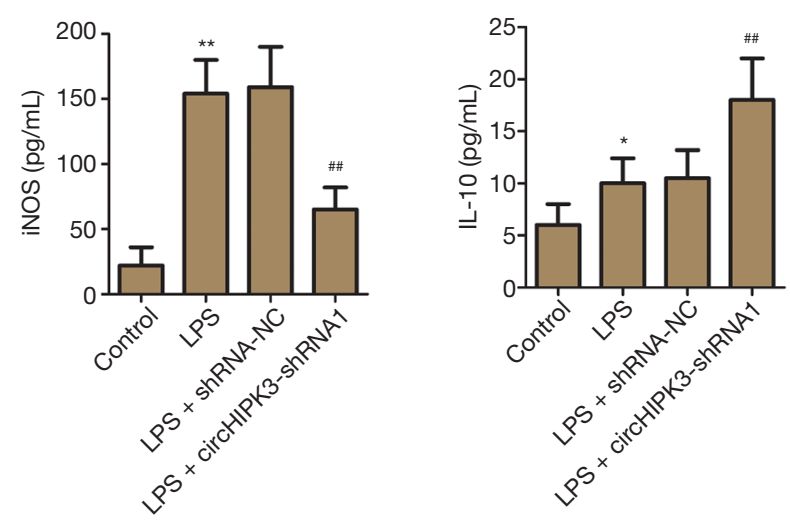

D
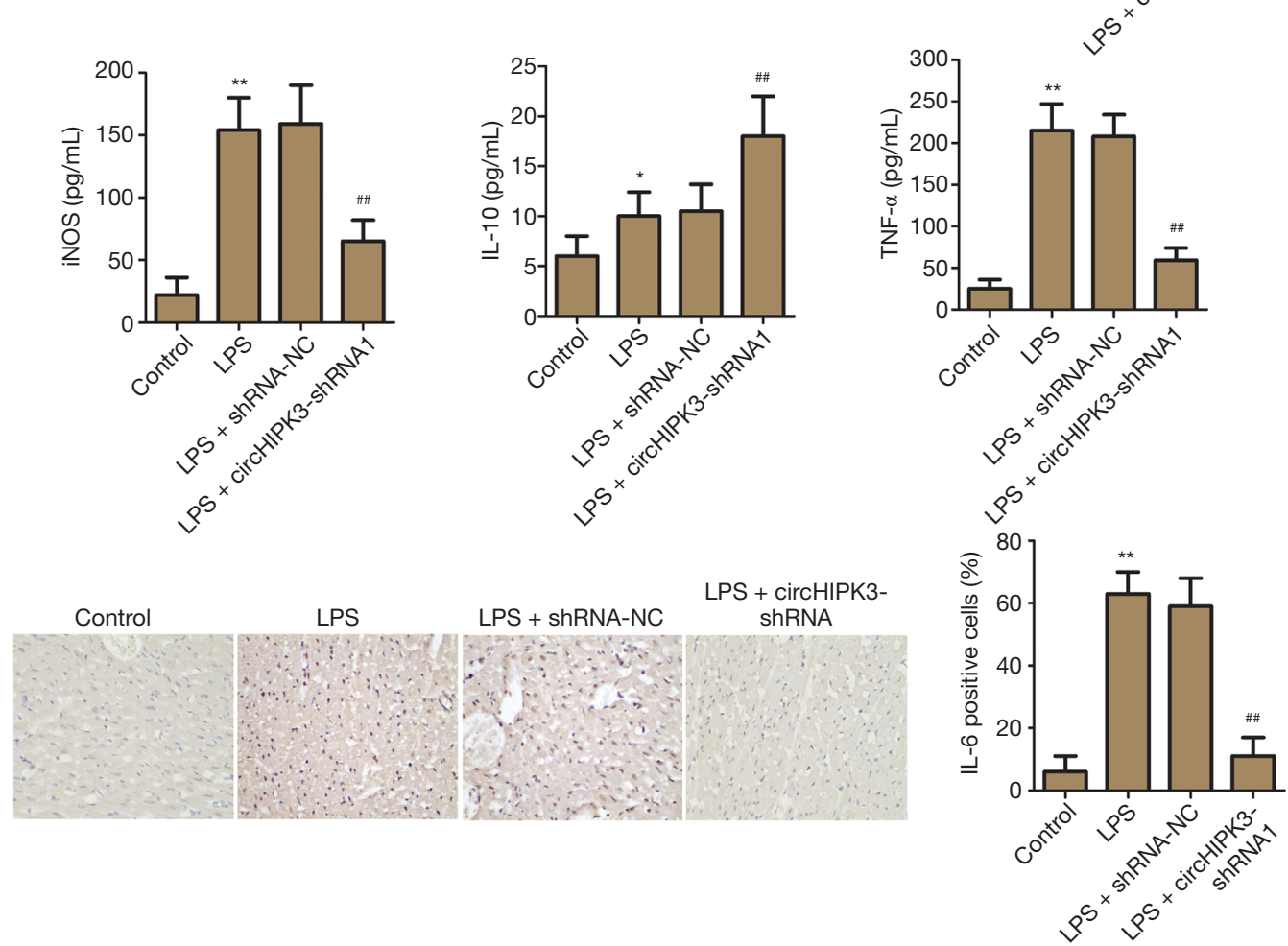

Figure 3 Effect of circHIPK3 knockdown on oxidative stress and inflammation in vivo. (A) Levels of oxidative stress markers: superoxide dismutase (SOD), malondialdehyde (MDA) and lactate dehydrogenase (LDH). (B) Effect of circHIPK3 knockdown on ROS generation. (C) Levels of inflammatory factors in peripheral blood: TNF- $\alpha$, iNOS, and IL-10. (D) Representative pictures of immunohistochemistry and percentage of IL-6 positive cells (magnification $\times 200$ ). The results are presented as mean \pm SD and represent three individual experiments $\left[^{*}\right.$, $\mathrm{P}<0.05,{ }^{* *}, \mathrm{P}<0.01$ versus control group; ${ }^{\#}, \mathrm{P}<0.01$ versus lipopolysaccharide (LPS)-induced model group]. 

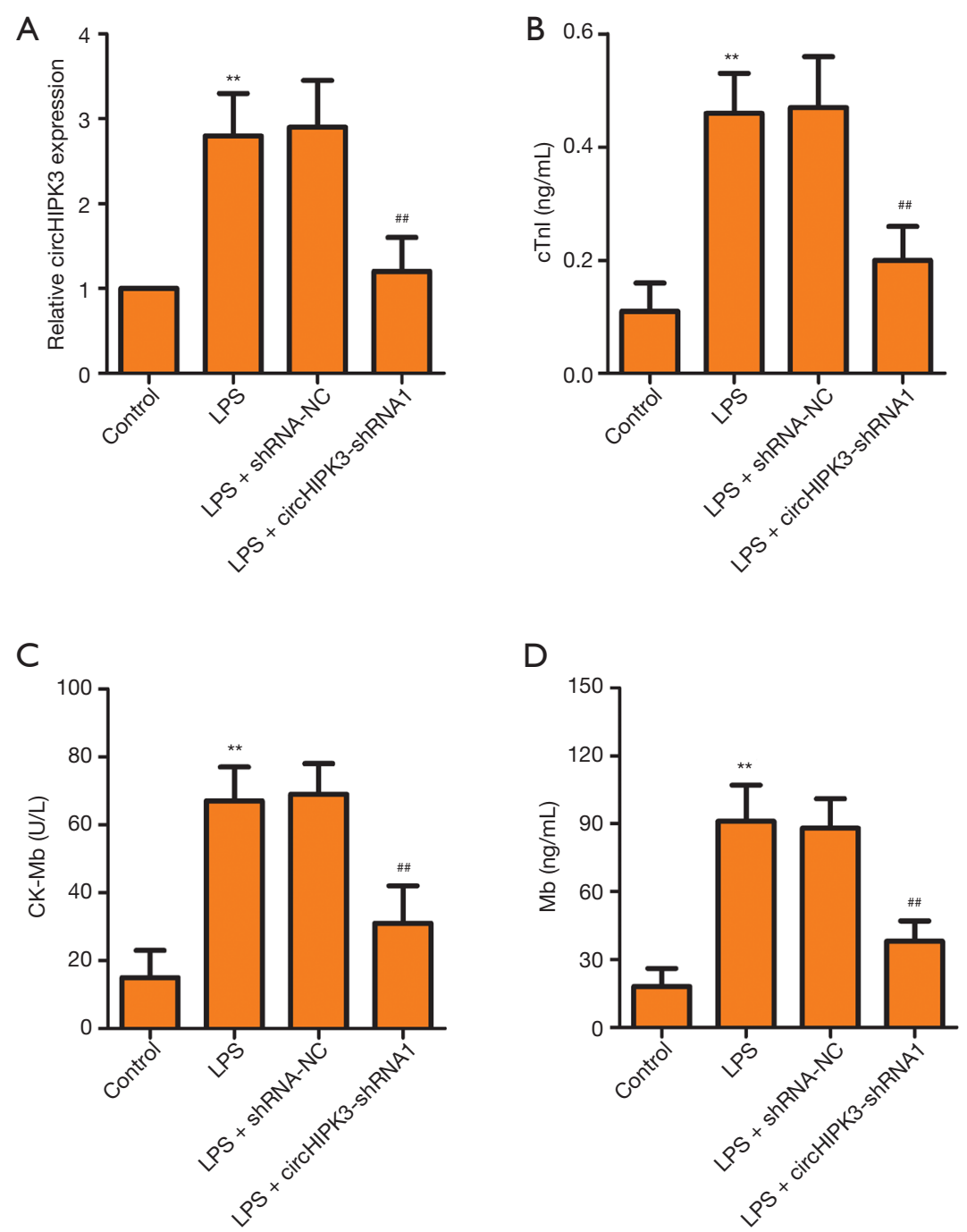

Figure 4 Effect of circHIPK3 knockdown on heart damage markers in H9C2 cells. (A) Relative circHIPK3 expression in H9C2 cells. (B) Expression level of cTnI. (C) Expression level of CK-MB. (D) Expression level of Mb. The results are presented as mean \pm SD and represent three individual experiments [** $\mathrm{P}<0.01$ versus control group; ${ }^{\# \#}, \mathrm{P}<0.01$ versus lipopolysaccharide (LPS)-induced model group].

function. It has been reported that LVSP was significantly decreased in CVB 3 induced myocarditis in golden hamsters (30), and HR was significantly reduced in autoimmune myocarditis (31). Further, H\&E staining revealed that knockdown of circHIPK 3 alleviated pathological damage in myocardial tissue that was induced by LPS. Myocardial enzymes are important markers of myocardial injury, and are released from damaged myocardial cells. Commonly used biomarkers for myocardial damage include CK-MB, cTnl, and Mb (32). In our study, knockdown of circHIPK3 remarkably restrained the release of CK-MB, cTnl, and Mb. Thus, it can be inferred from the results that knockdown of circHIPK3 can restore the contractile function of mice model cardiomyocytes.

Infiltration of leukocytes is a typical manifestation of pathological damage. In the pathological damage examination, histological examination results suggested that knockdown of circHIPK3 suppressed LPS-induced leukocyte infiltration. The infiltrating leukocytes always generate a variety of proinflammatory cytokines, such as TNF- $\alpha$, iNOS, and IL-6, which play vital roles in the progression of inflammation based myocardial damage (33). A previous study has shown that knockdown of circRNA 


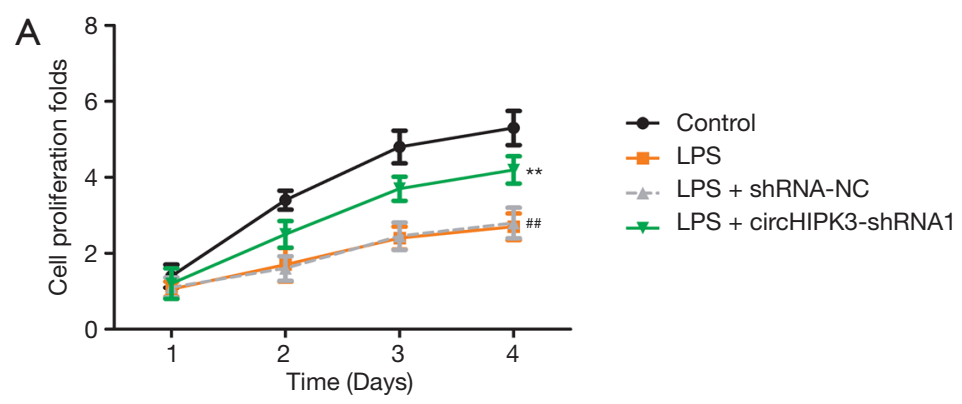

B
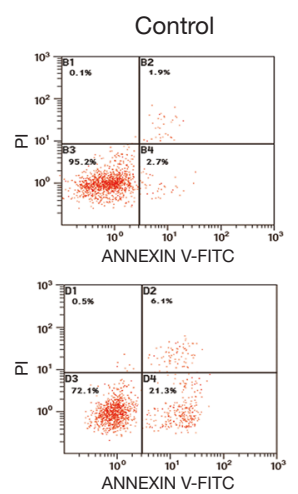

LPS + ShRNA-NC
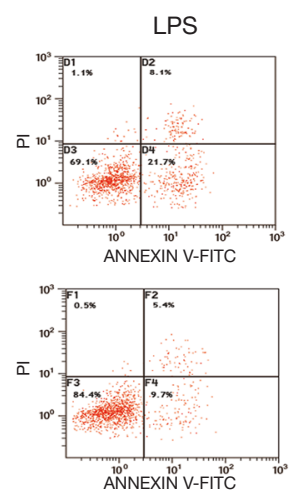

LPS + circHIPK3-shRNA1

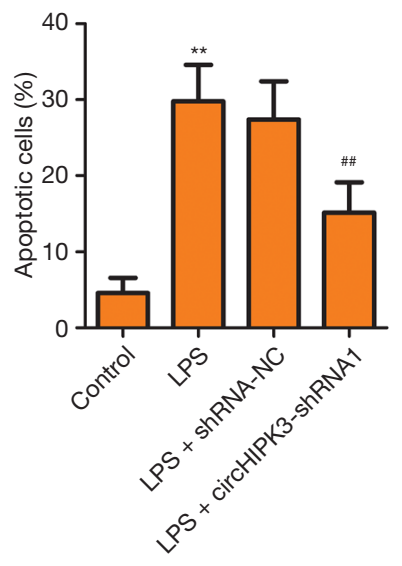

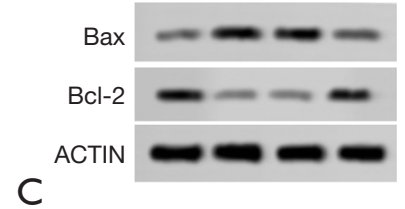

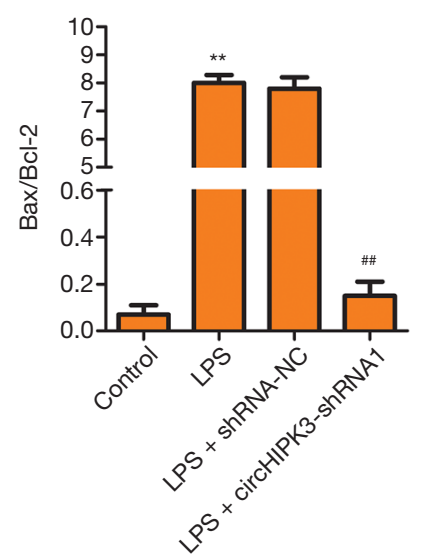

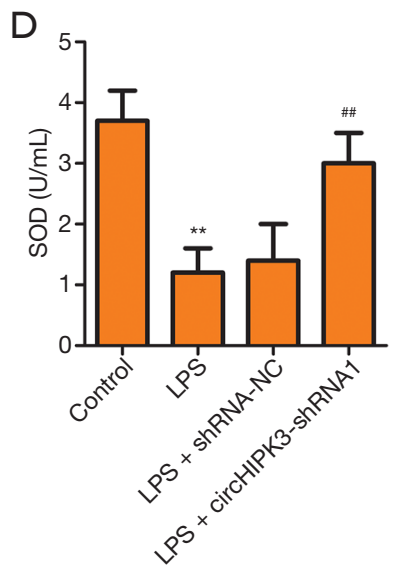
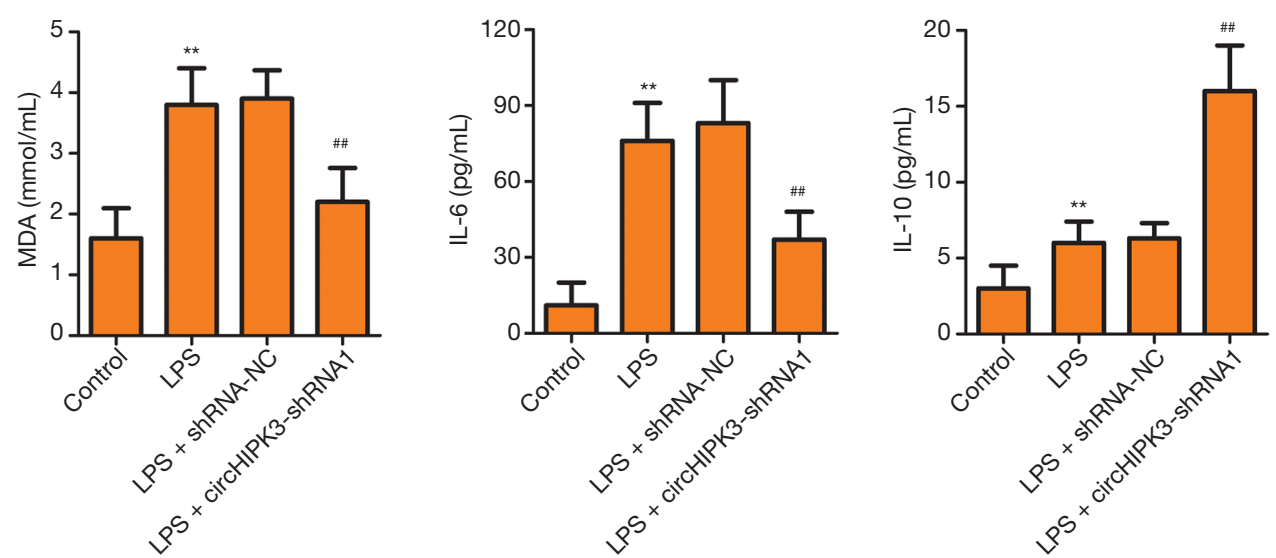

Figure 5 Effects of circHIPK3 knockdown on proliferation, apoptosis, oxidative stress, and inflammation of H9C2 cells. (A) Cell proliferation folds. (B) Representative pictures of flow cytometry and percentage of apoptotic cells. (C) Representative pictures of Western Blot assay and ratio of Bax/Bcl-2. (D) Levels of SOD and MDA. (E) Levels of IL-6 and IL-10. The results are presented as mean \pm SD and represent three individual experiments $\left[{ }^{* *}, \mathrm{P}<0.01\right.$ versus control group; ${ }^{\# \#}, \mathrm{P}<0.01$ versus lipopolysaccharide (LPS)-induced model group].

ANKRD36 could alleviate inflammatory injury in H9c2 cells exposed to LPS (14). In the present study, LPS induced upregulation of TNF- $\alpha$, IL-6, and iNOS in the heart was significantly inhibited by circHIPK3 knockdown. Oxidative stress is a basic protective mechanism regulating the normal activities of the human body, including proliferation, cell signal transduction and apoptosis (34). Chen et al. reported that circLRP6 regulated HG-induced proliferation, oxidative stress, and inflammation in mesangial cells via sponging miR-205, upregulating HMGB1, and activating 
the TLR4/NF- $\kappa B$ pathway. Our data consistently illustrated that knockdown of circHIPK3 treatment effectively suppressed the levels of ROS, MDA, and LDH release in model mice with LPS-induced myocarditis, also enhanced SOD level. These evidences revealed that the myocardial protection from circHIPK3 knockdown may be associated with inhibition of the myocardial inflammatory response and oxidative stress.

Caspases are the initiators and executors of apoptosis. Caspase- 3 and caspase-9 are critical apoptotic proteases, their function includes the regulation of cardiomyocytes, which play a pivotal role in the downstream of cascade junctions, and have a decisive role in the process of cell death $(35,36)$. As previously mentioned, $\mathrm{L}-\mathrm{N}^{\mathrm{G}}$-Nitro arginine methyl ester (L-NAME) attenuates cardiac myocyte apoptosis by inhibiting the expression of caspase- 3 , caspase-8, and caspase- 9 , thereby alleviating myocardial inflammation (37). Our data revealed that the expression of caspase-3, caspase- 9 , and the ratio of cleaved caspase-9/ caspase- 9 and cleaved caspase-3/caspase- 3 in protein level were significantly reduced by circRHIPK 3 knockdown. The ratio of $\mathrm{Bcl}-2 / \mathrm{Bax}$ is one of the critical indicators for characterizing apoptosis. When the rate of Bcl-2/Bax decreases, it suggests the occurrence of apoptosis (38). Shi et al. revealed that silencing circRNA ANKRD36 (sicircANKRD36) could alleviate inflammatory injury and apoptosis of H9c2 cells induced by LPS (14). Interestingly, knockdown of circHIPK3 significantly decreased the ratio of $\mathrm{Bax} / \mathrm{Bcl}-2$ in this study. These findings suggested that knockdown of circHIPK3 suppressed heart damage and cardiomyocyte apoptosis.

In this study, we focused on investigating the cardioprotective effects and apoptosis inhibition of circHIPK3 regulation on LPS-induced myocarditis in vivo and vitro. The results demonstrated that circHIPK3 can be upregulated significantly in LPS-induced myocarditis animal models and $\mathrm{H} 9 \mathrm{c} 2$ cells. In vivo, circHIPK3 knockdown effectively alleviated myocardial tissue damage, improved cardiac function and suppressed cardiomyocyte apoptosis. In vitro, circHIPK3 knockdown reversed the abnormalities mentioned that were induced by LPS. Therefore, we speculated that interference of circHIPK3 could mitigate the cardiac dysfunction in LPS-induced mice models and inhibit apoptosis in the H9C2 cardiomyocyte. The molecular mechanism may be related to some target miRNAs and sponging miRNAs. Additionally, the possible signaling pathways need to be explored further.

\section{Acknowledgments}

Funding: This study was supported by the Medical Science and Technology program and the health and Family Planning Commission program (2018020203) of Henan province and the department of science and technology program (182102310433) of Henan province.

\section{Footnote}

Reporting Checklist: The authors have completed the ARRIVE reporting checklist. Available at http://dx.doi. org/10.21037/atm-20-5306

Data Sharing Statement: Available at http://dx.doi. org/10.21037/atm-20-5306

Conflicts of Interest: All authors have completed the ICMJE uniform disclosure form (available at http://dx.doi. org/10.21037/atm-20-5306). The authors have no conflicts of interest to declare.

Etbical Statement: The authors are accountable for all aspects of the work in ensuring that questions related to the accuracy or integrity of any part of the work are appropriately investigated and resolved. All animal experiments were approved by the Animal Care and Use Committee of the Third Affiliated Hospital of Zhengzhou University (Zhengzhou, Henan, China) and conducted according to the declaration of the $8^{\text {th }}$ edition of the Guide for the Care and Use of Laboratory Animals (2011).

Open Access Statement: This is an Open Access article distributed in accordance with the Creative Commons Attribution-NonCommercial-NoDerivs 4.0 International License (CC BY-NC-ND 4.0), which permits the noncommercial replication and distribution of the article with the strict proviso that no changes or edits are made and the original work is properly cited (including links to both the formal publication through the relevant DOI and the license). See: https://creativecommons.org/licenses/by-nc-nd/4.0/.

\section{References}

1. Lv S, Rong J, Ren S, et al. Epidemiology and diagnosis of viral myocarditis. Hellenic J Cardiol 2013;54:382-91.

2. Pollack A, Kontorovich AR, Fuster V, et al. Viral myocarditis--diagnosis, treatment options, and current controversies. Nat Rev Cardiol 2015;12:670-80. 
3. Hou X, Fu M, Cheng B, et al. Galanthamine improves myocardial ischemia-reperfusion-induced cardiac dysfunction, endoplasmic reticulum stress-related apoptosis, and myocardial fibrosis by suppressing AMPK/ Nrf2 pathway in rats. Ann Transl Med 2019;7:634.

4. Ammirati E, Veronese G, Cipriani M, et al. Acute and Fulminant Myocarditis: a Pragmatic Clinical Approach to Diagnosis and Treatment. Curr Cardiol Rep 2018;20:114.

5. Kindermann I, Barth C, Mahfoud F, et al. Update on myocarditis. J Am Coll Cardiol 2012;59:779-92.

6. Moirangthem A, Patel T. Circular RNA: non-coding RNA with emerging roles in stem cell differentiation. Transl Cancer Res 2018;7:S50-2.

7. Szabo L, Salzman J. Detecting circular RNAs: bioinformatic and experimental challenges. Nat Rev Genet 2016;17:679-92.

8. Wang K, Long B, Liu F, et al. A circular RNA protects the heart from pathological hypertrophy and heart failure by targeting miR-223. Eur Heart J 2016;37:2602-11.

9. Xu H, Guo S, Li W, et al. The circular RNA Cdr1as, via miR-7 and its targets, regulates insulin transcription and secretion in islet cells. Sci Rep 2015;5:12453.

10. Ravnik-Glavač M, Glavač D. Editorial comments for the circular RNA circPRKCI promotion of tumor growth in lung adenocarcinoma. J Thorac Dis 2019;11:S1377-81..

11. Altesha MA, Ni T, Khan A, et al. Circular RNA in cardiovascular disease. J Cell Physiol 2019;234:5588-600.

12. Cai L, Qi B, Wu X, et al. Circular RNA Ttc3 regulates cardiac function after myocardial infarction by sponging miR-15b. J Mol Cell Cardiol 2019;130:10-22.

13. Zhang L, Han B, Wang J, et al. Differential expression profiles and functional analysis of circular RNAs in children with fulminant myocarditis. Epigenomics 2019;11:1129-41.

14. Shi S, Zhang S, Zhang H, et al. Silencing circANKRD36 protects $\mathrm{H} 9 \mathrm{c} 2$ cells against lipopolysaccharide-induced injury via up-regulating miR-138. Exp Mol Pathol 2019;111:104300.

15. Wang K, Gan TY, Li N, et al. Circular RNA mediates cardiomyocyte death via miRNA-dependent upregulation of MTP18 expression. Cell Death Differ 2017;24:1111-20.

16. Bei Y, Yang T, Wang L, et al. Circular RNAs as Potential Theranostics in the Cardiovascular System. Mol Ther Nucleic Acids 2018;13:407-18.

17. Yue F, Cheng Y, Breschi A, et al. A comparative encyclopedia of DNA elements in the mouse genome. Nature 2014;515:355-64.

18. Kai D, Yannian L, Yitian C, et al. Circular RNA HIPK3 promotes gallbladder cancer cell growth by sponging microRNA-124. Biochem Biophys Res Commun 2018;503:863-9.

19. Xiao-Long M, Kun-Peng Z, Chun-Lin Z. Circular RNA circ_HIPK3 is down-regulated and suppresses cell proliferation, migration and invasion in osteosarcoma. J Cancer 2018;9:1856-62.

20. Wu H, Dai A, Chen X, et al. Leonurine ameliorates the inflammatory responses in lipopolysaccharide-induced endometritis. Int Immunopharmacol 2018;61:156-61.

21. McGuire A, Brown JA, Malone C, et al. Effects of age on the detection and management of breast cancer. Cancers (Basel) 2015;7:908-29.

22. Lee NK, Choi YG, Baik JY, et al. A crucial role for reactive oxygen species in RANKL-induced osteoclast differentiation. Blood 2005;106:852-9.

23. Zhu JY, Yang X, Chen Y, et al. Curcumin Suppresses Lung Cancer Stem Cells via Inhibiting Wnt/ $\beta$-catenin and Sonic Hedgehog Pathways. Phytother Res 2017;31:680-8.

24. Ursu ON, Sauter M, Ettischer N, et al. Heme oxygenase-1 mediates oxidative stress and apoptosis in coxsackievirus B3-induced myocarditis. Cell Physiol Biochem 2014;33:52-66.

25. Sagar S, Liu PP, Cooper LT, Jr. Myocarditis. Lancet 2012;379:738-47.

26. Lukiw WJ. Circular RNA (circRNA) in Alzheimer's disease (AD). Front Genet 2013;4:307.

27. Bachmayr-Heyda A, Reiner AT, Auer K, et al. Correlation of circular RNA abundance with proliferation--exemplified with colorectal and ovarian cancer, idiopathic lung fibrosis, and normal human tissues. Sci Rep 2015;5:8057.

28. Li W, Zhong C, Jiao J, et al. Characterization of hsa_ circ_0004277 as a New Biomarker for Acute Myeloid Leukemia via Circular RNA Profile and Bioinformatics Analysis. Int J Mol Sci 2017;18:597.

29. Zhu X, Wang X, Wei S, et al. hsa_circ_0013958: a circular RNA and potential novel biomarker for lung adenocarcinoma. Febs j 2017;284:2170-82.

30. Morita H, Kitaura Y, Deguchi H, et al. Experimental coxsackie B3 virus myocarditis in golden hamsters. II. Evaluation of left ventricular function in intact in situ heart 14 months after inoculation. Jpn Circ J 1984;48:1097-106.

31. Gao S, Zhou J, Liu N, et al. Curcumin induces M2 macrophage polarization by secretion IL-4 and/or IL-13. J Mol Cell Cardiol 2015;85:131-9.

32. Zhen J, Li L, Yan J. Advances in biomarkers of myocardial injury in sepsis. Zhonghua Wei Zhong Bing Ji Jiu Yi Xue 2018;30:699-702. 
33. Gamkrelidze M, Intskirveli N, Vardosanidze K, et al. Myocardial dysfunction during septic shock (review). Georgian Med News 2014:40-6.

34. Ordonez FJ, Rosety MA, Camacho A, et al. Armcranking exercise reduced oxidative damage in adults with chronic spinal cord injury. Arch Phys Med Rehabil 2013;94:2336-41.

35. Liu M, Sun L, Jiang B, et al. Effect of nucleolin on cardiac cell apoptosis in Type 2 diabetic cardiomyopathy mice. Zhong Nan Da Xue Xue Bao Yi Xue Ban 2017;42:241-5.

36. Wang Q, Cui Y, Lin N, et al. Correlation of cardiomyocyte apoptosis with duration of hypertension, severity of

Cite this article as: Fan S, Hu K, Zhang D, Liu F. Interference of circRNA HIPK3 alleviates cardiac dysfunction in lipopolysaccharide-induced mice models and apoptosis in H9C2 cardiomyocytes. Ann Transl Med 2020;8(18):1147. doi: 10.21037/atm-20-5306 hypertension and caspase-3 expression in hypertensive rats. Exp Ther Med 2019;17:2741-5.

37. Ding GL, Han LN, Wang YT, et al. Study of L-NAME treatment on experimental autoimmune myocarditis. Zhongguo Ying Yong Sheng Li Xue Za Zhi 2013;29:119-23.

38. Um HD. Bcl-2 family proteins as regulators of cancer cell invasion and metastasis: a review focusing on mitochondrial respiration and reactive oxygen species. Oncotarget 2016;7:5193-203.

(English Language Editor: J. Jones) 